\title{
Imaging in influenza A encephalitis
}

\author{
S M Protheroe, D H Mellor
}

\begin{abstract}
Two cases of influenza $A$ encephalitis seen during an outbreak of influenza types $\mathbf{A}$ England/427/88 (H3N2) and A/Taiwan/1/86 (H1N1) in December 1989 are described. In both children the encephalitis developed within three days of the respiratory symptoms and both became comatose within 48 hours. Virological studies showed that the patients had had a recent influenza $A$ infection. Symmetrical localised hypodense lesions within the thalami and pons were demonstrated in both cases on computed tomography of the brain and striking findings in the pons in one case on magnetic resonance imaging. Influenza $A$ encephalitis is not easy to recognise clinically and serological confirmation can only be made after 10 days. Imaging may provide evidence in the acute stage to support a diagnosis of influenza encephalitis during influenza outbreaks.
\end{abstract}

An outbreak of influenza occurred in Nottinghamshire in late 1989. Two different influenza viruses A/England/427/88 (H3N2) A/Taiwan/1/ 86 (H1N1) were implicated in the epidemic. ${ }^{1}$ Two children admitted to our service during this period were found to have influenzal encephalitis. They showed unusual computed tomography of the head, which has been described previously in two cases of influenzal encephalitis. $^{2}$ Magnetic resonance imaging was carried out in our second case and showed striking changes.

\section{Case reports}

CASE 1

A 4 year old boy was admitted after two short generalised convulsions from which he had not gained full consciousness. He had been febrile the night before admission with malaise and cough. He had a history of febrile convulsions when aged 2.

On examination he was drowsy with a temperature of $38 \cdot 0^{\circ} \mathrm{C}$. His optic fundi were normal, but he had nystagmus and was hypertonic with brisk deep tendon reflexes and extensor plantar responses. There was no meningeal irritation or sensory disturbance. He had a further fit and remained drowsy.

Cerebrospinal fluid showed a normal protein concentration $(0.3 \mathrm{~g} / \mathrm{l})$ with no increase in cells. Full blood count, blood glucose, urea and electrolytes, hepatic enzymes, copper, caeruloplasmin, lactate, ammonia, and coagulation tests were all normal. Urine amino acids and organic acids were also normal. Electroencephalography showed generalised slow wave activity.

Computed tomography of the brain on the second day showed low attenuation changes in the thalami bilaterally (fig 1). There was some brainstem swelling with low attenuation change in the pons (fig 2). Both putamina and globus

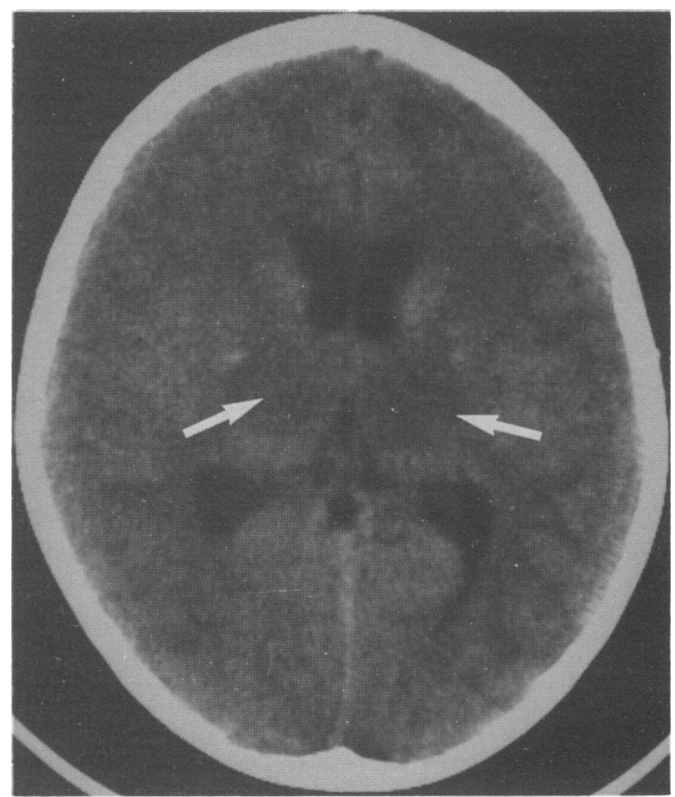

Figure 1 Axial computed tomogram of the brain in case 1 showing low attenuation lesions in both thalami (arrowed).

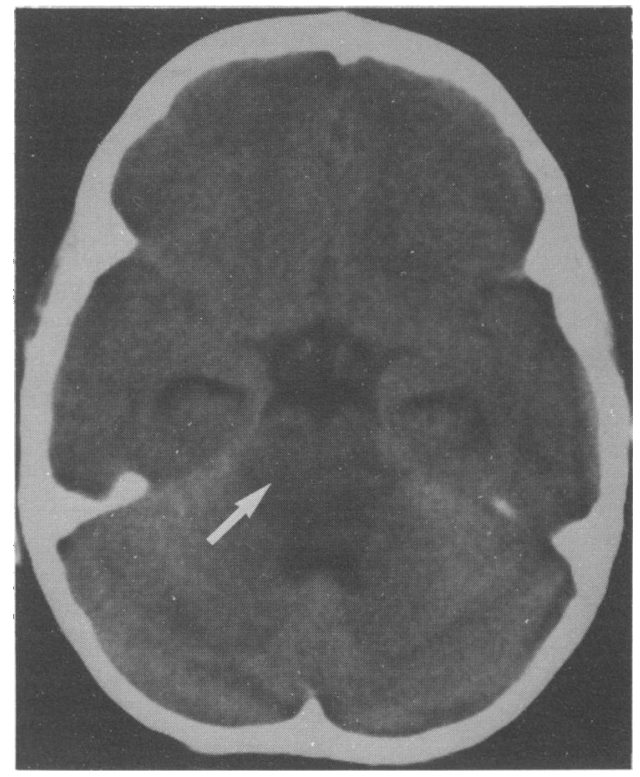

Figure 2 Axial computed tomogram of the brain in case 1 showing swelling and low attenuation change in the pons (arrowed). 
pallidi showed small foci of high attenuation possibly representing haemorrhage.

On the third day his condition deteriorated with decorticate posturing, early papilloedema, and a sixth nerve palsy on the right. Dexamethasone was started, he was ventilated, and an intracranial pressure monitoring device was inserted. He was treated with acyclovir and thiamine, although the features were not typical or herpes simplex encephalitis or Leigh's disease.

He was extubated after four days but remained in a somnolent state. By day 10 he had begun to improve, although he showed choreoathetoid movements of his trunk and limbs on purposeful movement. Complement fixation titre for influenza $A$ virus was less than 16 on admission, but increased to +256 on the 15 th day, indicating recent influenzal infection. Serological tests failed to demonstrate any other virus antibody response and we were not able to find any other cause for coma or encephalopathy.

He was discharged on day 28 , walking with an aid and speaking in sentences. Follow up three months later showed he had made a full neurological and intellectual recovery apart from a persisting right lateral rectus palsy.

CASE 2

An 11 year old girl was admitted having been found semicomatose by her parents. For the previous three days she had been febrile with headache, cough, and malaise. Two family members also had influenza like symptoms. She had had an acute encephalitis at age 3 , from which she had recovered, but had been left with residual behavioural problems, left divergent squint, precocious puberty, and mild optic atrophy. This episode had also followed an upper respiratory tract infection and influenza $A$ was isolated from a throat swab. Computed tomography of the brain at that time had shown linear hypodensities in the external capsule region on both sides.

On this occasion she was drowsy but able to respond to commands on admission. Pale optic discs were noted as before. She was pyrexial and had brisk tendon reflexes with extensor plantar responses. The cerebrospinal fluid showed a raised protein concentration $(1 \cdot 2 \mathrm{~g} / \mathrm{l})$ with no white cells, $36 \mathrm{red}$ cells $/ \mathrm{mm}^{3}$, and normal glucose. Serum electrolytes, ammonia, hepatic function tests, copper, caeruloplasmin, lactate, and a full blood count were within normal limits, as were urine amino acids and organic acids. Trichrome staining of a muscle biopsy specimen showed no 'ragged red muscle fibres'.

She became unrousable by the second day and was unresponsive to pain with loss of the gag reflex. Central respiratory irregularities, namely hyperventilation and Cheyne-Stokes respiration became apparent, and she was intubated and ventilated. She was treated with acyclovir and dexamethasone.

Computed tomography of the brain showed symmetrical well defined areas of low attenuation in both thalami extending to involve the capsular fibres. Further diffuse low attenuation changes were seen within the pons and midbrain. Some swelling of the brainstem was seen. Low attenuation changes considered longstanding and a result of her previous encephalitis were noted in the external capsules. Magnetic resonance imaging inversion recovery sequences of the brain showed low signal changes in the thalami and pons (fig 3). Axial T2 weighted images showed ring like areas of high signal intensity with central low signal in the pons (fig 4). Complement fixation titres for influenza $A$ virus were less than 16 on admission but increased to +256 by day 15 .

By day 17 of her illness it was possible to take her off the ventilator and extubate her. She opened her eyes to sensory stimuli but did not recognise her parents. Spasticity affected all four limbs; she was unable to swallow and required tube feeding. She remained in this 'sleeping beauty, akinetic mute' state for three months without much change. Progress was very slow and five months after the onset of her illness she was still immobile with expressionless facies and paucity of movement. She could sit in a chair with support and recognise her

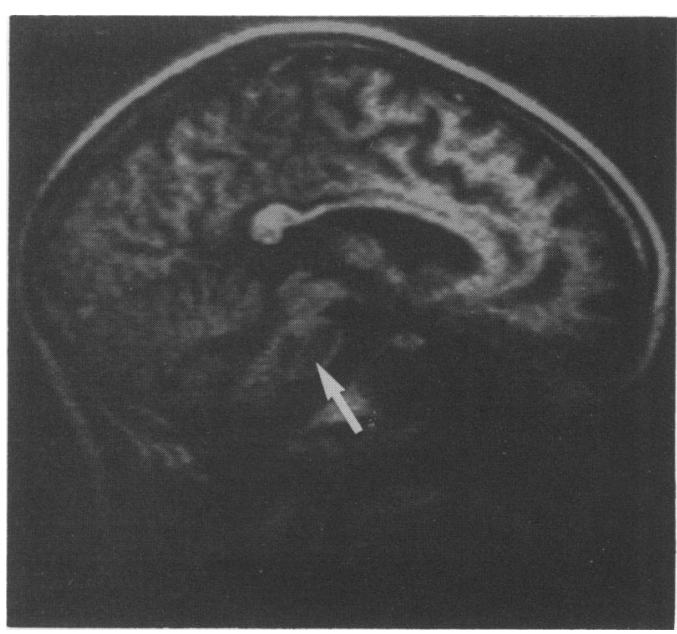

Figure 3 Saggital inversion recovery magnetic resonance imaging brain scan in case 2 showing low signal changes in the pons (arrowed).

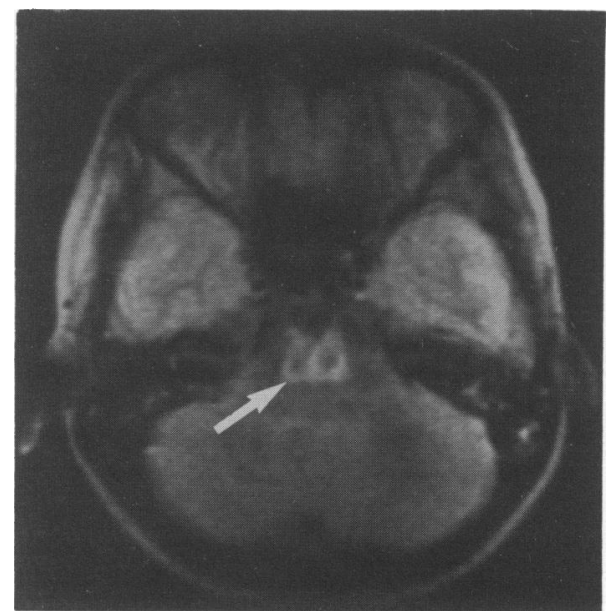

Figure 4 Axial T2 weighted magnetic resonance imaging brain scan in case 2 showing ring like areas of high signal in the pons (arrowed). 
parents. Speech had not returned but she could use a simple communication board and understand simple sentences.

\section{Discussion}

These two children were considered to have influenza A encephalitis on the basis of the prodromal illness, clinical course, and virology studies. Clinically they resemble the six cases described by Delorme and Middleton, who developed fever, reduced consciousness, and neurological signs after influenzal symptoms. ${ }^{4}$ Analyses of the cerebrospinal fluid were normal in these six cases apart from a slight increase in protein in one patient, but computed tomography was not done.

True encephalitis is comparatively rare after influenza A but benign confusional states and minor neurological signs (for example, ocular muscle palsies) are observed more often. ${ }^{5}$ Parainfectious processes such as transverse myelitis and Reye's syndrome are also observed, ,-10 although virus isolation from the brain or cerebrospinal fluid is infrequent. ${ }^{11} 12$ Neurological symptoms and signs may appear several weeks after the infection making it difficult to establish an aetiological link in some cases.

The finding of focal low attenuation lesions in the frontotemporal regions on computed tomography can be helpful in the diagnosis of herpes simplex encephalitis. ${ }^{13} 14$ Computed tomography is usually diagnostically unrewarding in other forms of encephalitis. However, our findings suggest that there may be a typical appearance on computed tomography in influenza encephalitis, and this is in keeping with two reports from Japan describing similar localised hypodense lesions. ${ }^{2}{ }^{3}$ Recognition of these tomographic abnormalities in patients with possible influenzal encephalitis may aid diagnosis while viral titres are awaited.

The findings on magnetic resonance imaging in brainstem encephalitis include pontine enlargement and increased signal lesions in the pons. ${ }^{15}{ }^{16}$ Magnetic resonance imaging is superior to computed tomography for detecting brainstem encephalitis, as seen in our second case where obvious pontine abnormalities were shown on magnetic resonance imaging but not well seen on computed tomography. A similar discrepancy between magnetic resonance imaging and tomography findings in brainstem encephalitis has been previously reported where discrete abnormal areas were seen in the pons, midbrain, and thalamus on magnetic resonance imaging but the computed tomogram had revealed only a low density area in the pons. ${ }^{17}$

It has been observed that a normal computed tomogram on follow up correlates with full clinical recovery in viral encephalitis in general. ${ }^{18}{ }^{19}$ This is also seen in the previously reported case of influenza $A$ encephalitis where the initial abnormal hypodense areas in the midbrain, basal ganglia, and cerebral white matter had disappeared on follow up computed tomography and the patient made a good recovery. ${ }^{3}$

The cause of these lesions is unknown. It has been postulated that hypodense areas on computed tomography in encephalitis reflect the sequelae of ischaemia induced by compression of end arteries by brain oedema or may be due to localised areas of inflammation. It is known that the virus can have a direct cytopathic effect on vulnerable sites. ${ }^{27}$

Our second case experienced two episodes of acute encephalitis when infected with influenza A, which could not be explained by any other identifiable cause. On both occasions there was virological evidence of recent influenza infection. The original episode was associated with bilateral hypodense areas in the external capsule regions on computed tomography, with low attenuation lesions in both thalami, pons, and midbrain on the second scan.

Localised hypodense lesions in the basal ganglia have also been described on computed tomography in encephalitis after infections with ECHO, mumps, measles, and Coxsackie viruses. ${ }^{18} 2021$ Other causes of low density areas in the basal ganglia and thalamus have been described in disorders such as Leigh's encephalopathy, Wilson's disease, and mitochondrial cytopathies as well as carbon monoxide poisoning and anoxia. ${ }^{22}$ Wilson's disease, Leigh's encephalopathy, and mitochondrial cytopathies seem unlikely in view of the normal copper studies and lactate concentrations in both cases and the normal muscle biopsy specimen in case 2 .

Circumstantial evidence has implicated the influenza virus as the cause of the encephalitis lethargica epidemic of the $1920 \mathrm{~s}^{23}$ Similar isolated cases have been described from year to year often at the time of influenza epidemics. ${ }^{24-29}$ von Economo first described the illness, postulated a viral aetiology, and reported foci of inflammation in the grey matter of the midbrain and basal ganglia. ${ }^{30}$ The manifestations of encephalitis lethargica were variable but a number of clinical features have been proposed as major criteria to support the diagnosis. ${ }^{31}$ Our first case showed signs of basal ganglia involvement with choreoathetoid movements, ophthalmoplegia, and somnolence. Our second case exhibited akinetic mutism, central respiratory irregularities, and somnolence. Combined with the acute encephalitic illness, our cases fulfil the criteria to support the diagnosis of encephalitis lethargica. We are aware that unexpected neurological sequelae such as parkinsonism with oculogyric crises can appear after apparent recovery as long as four years later.

1 PHLS Communicable Disease Surveillance Centre. Com municable disease report. CDR 89/44. London: CDSC, 1989; 3rd November: $3-4$.

2 Hattori H, Kawamori J, Takao T, et al. Computed tomography in postinfluenzal encephalitis. Brain Dev 1983;5: graphy

3 Okuno T, Takao T, Ito $M$, Mikawa $H$, Nakano Y. Contrast enhanced hypodense areas in a case of acute disseminated encephalitis following influenza A virus. Comput Radiol encephalitis follov

4 Delorme L, Middleton PJ. Influenza A virus associated with acute encephalopathy. Am $\mathcal{F}$ Dis Child 1979;13:822-4.

5 Sulkava R, Rissanen A, Pyhala R. Post-influenza encephalitis during the influenza A outbreak in 1979/80. F Neurol Neurosurg Psychiatry 1981;44:161-3

6 Wells CEC. Neurological complications of so-called 'influenza' winter study in S E Wales. BMF 1971;i:369-73.

7 Booss J, Esiri M. Epidemic encephalitis I. Viral encephalitis Pathology, diagnosis and treatment. Oxford: Blackwell Scientific Publications, 1986:141.

8 Flewett TH. Influenzal encephalopathy and post-influenzal encephalitis. Lancet 1958;ii:11-5.

9 Dubowitz V. Influenzal encephalitis. Lancet 1958;i:140. 
10 Horner FA. Neurological disorders after Asian intluenza. N Englf Med 1958;258:983-5.

11 Anonymous. News and Notes. Epidemiology. Laboratory Reports. BMF 1970;i:311

12 Frankova V, Jirasek A, Tumova B. Type A influenza virus isolations from different organs in human lethargica cases. Arch Virol 1977;53:265-8.

13 Thompson JLG. The computed axial tomography in acute herpes simplex encephalitis. Br F Radiol 1976;49:86-7.

14 Davis JM, Davis KR, Leinman GM, Kirchner HS, Taveras JM. Computed tomography of herpes simplex encephalitis with clinicopathological correlation. Radiology 1978;129: with clinicc 17 .

15 Davidson HD, Steiner RE. Magnetic resonance imaging in infections of the central nervous system $A \mathcal{F} N R \quad 1985 ; 6$ : infection

16 Furman JM, Brownstone PH, Baloh RN. A typical brainstem encephalitis magnetic resonance imaging and oculographic features. Neurology 1985;35:438

17 Hosoda K, Tamaki N, Masumoa M, Matsumoto S. Magnetic resonance images of brainstem encephalitis. $\mathcal{f}$ Neurosurg 1987;66:283-5.

18 Charney R, Orecchio EJ, Simmerman RA, Berman RH Computerised tomography in infantile encephalitis. $A m$ f Dis Child 1979;133:803-5.

19 Bittner T, Dorndorf W. Prognostic value of computerised tomography and cerebrospinal fluid analysis in vira encephalitis. F Neuroimmunol 1988;20:16.

20 Goutieres F, Aicardi J. Acute neurological dysfunction associated with destructive lesion of the basal ganglia in children. Ann Neurol 1982;12:328-32.

21 Ochi J, Okuno T, Uenoyama Y, Narita H, Mikawa H. Symmetrical low density areas in bilateral thalami in an infant with measles encephalitis. Comput Radiol 1986;10: infant with.

22 Aicardi J, Gordon N, Hagberg B. Med Child Neurol 1985;25:249-60. (encephaliti Inhuenza caused epidemic encephalitis (encephalitis lethargica). The circumstantial evidence and challenge to the naysayers. Med Hypotheses 1989;28:18.

24 Leigh AD. Infections of the nervous system occurring during an epidemic of influenza B. BMF 1946;ii: 936 .

25 Espir MLE, Spalding JMK. Three recent cases of encephalitis lethargica. $B M \mathcal{F}$ 1956;i:1141-4.

26 Hunter R, Jones M. Acute lethargic type encephalitis. Lance 1966;ii: $1023-4$.

27 Anonymous. Encephalitis of lethargica type in a mental hospital [Editorial]. Lancet 1966;ii:1014-5.

28 Greenough A, Davis JA. Encephalitis lethargica. Mystery of the past or undiagnosed disease of the past? Lancet 1983; $\mathbf{i}$ : the past

29 Anonymous. Encephalitis lethargica [Editorial]. Lancet 1981; ii: 1396 .

30 von Economo C. Encephalitis lethargica: its sequelae and treatment. Translated by KO Newman. London: Oxford Uniress, 1931.

31 Howard RS, Lees AJ. Encephalitis lethargica. A report of four recent cases. Brain 1987;110:19-33.

\section{Addendum}

Since this paper was accepted for publication case 1 was readmitted to hospital after a seizure at home. Vomiting and a further seizure occurred after admission. He was drowsy and febrile but there was no neck stiffness and no focal neurological findings. Blood urea, electrolytes, glucose, prothrombin time, liver function tests, haemoglobin, and platelets were normal. Total peripheral white cell count was low at $2 \cdot 2 \times 10^{9} / 1$.

He was given an intravenous loading dose of phenytoin. Computed tomography showed no changes. The cerebrospinal fluid was sampled and contained one lymphocyte $/ \mathrm{mm}^{3}$, a normal glucose, but a raised protein concentration of $1.6 \mathrm{~g} / \mathrm{l}$, and a pressure of $35 \mathrm{~cm} \mathrm{H}_{2} \mathrm{O}$. Further short seizures occurred and then a respiratory arrest requiring resuscitation and mechanical ventilation. Despite passive hyperventilation and mannitol his pupils remained fixed and dilated. Brainstem function tests showed no activity and ventilatory support was withdrawn after a period of 12 hours. Permission for postmortem examination was not given. Subsequently the results of viral antibody titres sent on admission became available. All were negative including influenza $A$ and $B$.

\section{COMMENT}

The previous encephalitis had occurred in association with a serologically proved influenza infection. This fatal illness a year later raises the possibility that the original illness could have been some kind of metabolic encephalopathy precipitated by the influenza, although detailed metabolic investigations were negative. 